\title{
Coagulopathy in COVID-19 from Pathogenesis until Treatment: A systemic Review
}

\author{
Nasser Mousa ${ }^{1}$, Ahmed Abdel-Razik ${ }^{1}$, Eman Mousa ${ }^{2, *}$, Tasnim Elbadrawy ${ }^{3}$, Khaled Hosni $^{3}$, Aya Mousa ${ }^{4}$, Ahmed \\ Taham $^{3}$, Alaa Elmetwalli ${ }^{5}$ \\ ${ }^{1}$ Tropical Medicine Department, Mansoura University, Mansoura, Egypt; ${ }^{2}$ Faculty of Dentistry, Mansoura University, \\ Mansoura, Egypt; ${ }^{3}$ Faculty of Medicine, October 6 University, Egypt; ${ }^{4}$ Faculty of physical therapy, Galala university, Egypt; \\ ${ }^{5}$ Department of Clinical Trial Research Unit, Egyptian Liver Research Institute and Hospital, Mansoura, Egypt.
}

\begin{abstract}
Background: Nowadays, the new coronavirus-induced severe acute respiratory syndrome (COVID-19) outbreak was primarily stated in December 2019. Three months later, the Director-General of the World Health Organization, avowed the COVID-19 a global pandemic. Gathered evidence discloses that coagulation syndrome is repeatedly seen in COVID-19, and the incidence is higher in severe cases. The worsening lung functions and coagulopathy are some of the most significant poor prognostic criteria in the outcome of COVID-19. The pragmatic coagulopathy in patients hospitalized with COVID-19 (COVID-19 associated coagulopathy) is categorized by the increased level of D-dimer and fibrinogen/fibrin degradation products. They are interrelated with the severity of COVID19. This elevation will aid the arrangement of the patients consistent with the disease severity and management. These coagulation parameters elevation can point to a poor prognosis. Coagulopathy in COVID-19 mainly affects immobilized patients, those in critical care units, and those who have extra risk factors that predispose to coagulopathy, for instance, acute inflammatory conditions and hypoxia. The exact pathogenesis of hypercoagulability in COVID-19 is unwell understood. The signs suggest that the COVID-19 coagulopathy is an evolving phenomenon in which endothelial damage, platelet aggregation, augmented innate immune response, and other measures as systemic inflammation favoring coagulation would divide up the severity of this disease. Understanding the pathophysiology of COVID-19 related coagulopathy is crucial for suitable treatment and monitoring of these patients. Encouraging interest in the potential uses of anticoagulation in COVID19 particularly is the use of heparin that has a potential benefit over other anticoagulants due to its anticoagulant role, anti-inflammatory, and potentially anti-viral properties.
\end{abstract}

\section{Introduction}

Generally speaking, In December 2019, an emerging virus named severe acute respiratory syndrome

Keywords: COVID-19; coagulopathy; endothelial dysfunction; Ddimer

Received: 3-3-2021; Accepted: 11-3-2021

* Corresponding author. Email: Eman.nasser.mousa @yahoo.com coronavirus-2 (SARS-CoV-2) was isolated in patients with pneumonia. The disease caused by this virus is called coronavirus disease-19 (COVID-19). The WHO declared the COVID-19 outbreak a global health emergency ${ }^{1}$. Even though SARS-CoV-2 is documented to produce substantial pulmonary disease, including pneumonia and acute respiratory distress syndrome (ARDS), clinicians have noticed many extrapulmonary manifestations of COVID-19 together with, hematological, cardiovascular, renal, gastrointestinal, and hepatobiliary, endocrinologic, neuropsychiatric, ophthalmologic, and dermatologic manifestations ${ }^{2-4}$.

Characteristically, the virus produces a functional disturbance in cell receptors of angiotensin-converting enzyme 2 (ACE2). These receptors are present in human cells including, type II alveolar cells, gastrointestinal cells, myocardial cells, and renal cells. Angiotensin-converting enzyme- 2 receptors allow the entry of the virus into the cell and have been confirmed to be the cell receptor of SARS$\mathrm{CoV}-2$. Downregulation of ACE-2 by SARS-CoV-2 infection producing increased pathological consequences of COVID-19 ${ }^{5,6}$. The principal coagulation abnormalities in patients with COVID-19 propose a hypercoagulable state, which has been entitled thrombo-inflammation or COVID19-associated hemostatic abnormalities 7,8. Recently, Cytokine storm is a predominant feature in coronaviruses. COVID-19 infects the endothelial cells and gives off a strong local inflammatory reaction that is an essential cause for the development of a systemic inflammatory response and cytokine storm ${ }^{9}$. Furthermore, key features of a cytokine storm syndrome are inflammatory effects of activated vascular endothelial cells, and endothelial injury with resultant prothrombotic properties. Vascular endothelial injury causes additional thrombocytopenia, reduction of natural anticoagulants, and also hemostatic activation ${ }^{10}$.

The incidence of thrombotic phenomena associated with COVID-19.

Importantly, laboratory and imaging studies found an increased risk of thrombotic complications in patients with SARS-CoV-2 infection. Klok et al assessed the incidence of thrombotic complications in COVID-19 patients admitted to the ICU. They found that $31 \%$ of these patients had thrombotic complications, even in patients who were already getting standard doses of prophylactic 
anticoagulation 11. The incidence of disseminated intravascular coagulopathy (DIC) was found as $71.4 \%$ in Chinese patients diagnosed with COVID-19 pneumonia ${ }^{12}$. Patients with COVID-19 pneumonia similarly have a high risk of pulmonary embolism. Rising levels of d-dimer help the diagnosis of a thromboembolic patient. Out of 1008 hospitalized patients, 25 with confirmed pneumonia underwent computed tomography pulmonary angiography.

Pulmonary embolism was diagnosed in $40 \%$, mainly localized in small branches of the pulmonary artery. Remarkably, these patients presented significantly higher median d-dimer levels (11.07 $\mu \mathrm{g} / \mathrm{mL}$; IQR: 7.12) compared to median d-dimer levels of $2.44 \mu \mathrm{g} / \mathrm{mL}$ (IQR: 1.68) in patients without pulmonary emboli (PE) ${ }^{13}$. It has been theorized that thrombi may play a substantial role in gas exchange irregularities and multisystem organ dysfunction in COVID-19 pneumonia ${ }^{14}$. It has appeared that in a singlecenter retrospective study, 81 patients with COVID-19 were categorized who had to be admitted to an intensive care unit and the incidence of deep vein thrombosis was determined. The patients did not receive thromboprophylaxis. Twentyfive patients $(25 \%)$ had deep vein thrombosis (DVT) of the lower extremities, from which $40 \%$ of whom died ${ }^{15}$. Likewise, autopsy studies postulate the incidence of pulmonary endothelial injury and micro thrombosis, four autopsies of SARS-CoV-2 infected patients from New Orleans with sudden respiratory decompensation, small thrombi were present in sections of the peripheral lung parenchyma. Besides, the microscopic findings confirmed that small vessels confined thromboembolisms and small thrombi together with scattered areas of diffuse alveolar damage, demonstrating that small vessels can be exaggerated by micro thrombosis ${ }^{16}$.

\section{Coagulation aberrations in COVID-19}

Abundant coagulation abnormalities were originated in COVID-19 involved, endothelial dysfunction and cytokine storm, excess thrombin generation, and fibrinolysis shutdown ${ }^{17}$. The initial coagulopathy of COVID-19 has been presented as increased D-dimer and fibrinogen or fibrin degradation products over prothrombin time prolongation and thrombocytopenia. Among the nonsurvivors, the maximum score in the DIC parameter was in the D-dimer measurements $(85.7 \%$ compared to $23.8 \%$ in platelet count, $28.6 \%$ in fibrinogen, and $47.6 \%$ in PT). This observation suggested that secondary hyperfibrinolysis following the coagulation activation played a dominant role in the COVID-19-associated coagulopathy ${ }^{18}$. Up-to-date guidelines from different countries suggest that coagulation markers at hospital and intensive care unit (ICU) admission should include; D-dimers, PT and/or an international normalized ratio (INR), aPTT, platelet count, and fibrinogen to measure daily or twice daily depending on the initial results. Twice-daily monitoring should be considered in case of altered coagulation parameters at first look (platelet count $<100 \times 10^{9} / \mathrm{L}$, fibrinogen $<2 \mathrm{~g} / \mathrm{L}$, and raised D-dimer (although a specific cutoff for D-dimer cannot be defined, a three to four-fold elevation is considered a markedly raised value) ${ }^{19}$.
In this way, a study was conducted in Spin has revealed that higher D-dimer levels were accompanied by a greater incidence of pulmonary embolism 3, 6, 9, and 12 days after determining D-dimer levels with of 1.7, 2.0, 2.4, and 2.4, respectively in 21 patients from Spain ${ }^{20}$. Zhou et al ${ }^{17}$ disclosed in a multivariable regression analysis, an increased odds of hospital death associated with older age and D-dimer $>1 \mu \mathrm{g} / \mathrm{mL}$ on hospital admission. Furthermore, a high D-dimer at admission was an independent predictor for mortality in COVID-19 patients where are patients with a D-dimer $\geq 2.0 \mu \mathrm{g} / \mathrm{ml}$ had much higher mortality versus those with levels $\leq 2.0 \mu \mathrm{g} / \mathrm{ml}^{21}$. Consequently, the potential risk factors of older age and D-dimer $\geq 2 \mu \mathrm{g} / \mathrm{mL}$ may help clinicians to classify patients with poor prognoses at an early stage. Conspicuously, Tang et al., ${ }^{22}$ conveyed that patients with D-dimer $>3 \mu \mathrm{g} / \mathrm{mL}$ (6-fold of the upper limit of normal) showed a significant decrease in 28-day mortality $(32.8 \%$ vs. $52.4 \% ; P=.017)$ if treated with unfractionated heparin (UFH) or low molecular weight heparin $(\mathrm{LMWH})^{23}$. Accordingly, D-dimer may be able to predict which COVID-19 patients have poorer outcomes. This could have a significant impression on the ability of clinicians to figure out which patients are likely to have worse prognoses and act prophylactically 24,25 .

\section{Pathogenesis of coagulation abnormalities in COVID-} 19.

Illustratively, there is now evidence that some patients may respond to COVID-19 with an exuberant "cytokine storm" response ${ }^{26}$. Findings from the SARS epidemic provide possible viral-specific mechanisms for acute respiratory distress syndrome (ARDS) and uncontrolled coagulation. Past Autopsy studies of patients who died of SARS pneumonia identified the SARS-CoV spike (S) protein in cells expressing the receptor angiotensinconverting enzyme-2 (ACE2) ${ }^{27-30}$. On one hand, the main candidate receptor for SARS-CoV-2 ${ }^{31,32}$ is the binding of the $\mathrm{S}$ protein to ACE2 receptors on endothelial cells that initiates localized inflammation, endothelial activation, tissue damage, and altered release of proinflammatory cytokines including tumor necrosis factor (TNF) and interleukins, including interleukin 1( IL-1) and interleukin 6 (IL-6). The IL-6 must induce the expression of tissue factor in macrophages, followed by the activation of coagulation and generation of thrombin, which has been concerned in thrombogenesis ${ }^{33}$. On the other hand, angiotensin-II (AngII), which is metabolized by ACE-2 to the vasodilatory and anti-inflammatory peptide angiotensin has an imperative role in this phenomenon. The consumption of ACE-2 by the viral entry in the early phases of the infection leads to the interruption of AngII metabolism leading to an upsurge in its plasma concentration. AngII exerts several prothrombotic effects, including vasoconstriction, endothelial and platelet activation, along with pro-inflammatory-cytokine release 34. While these changes appear to be predominantly in the lungs, endothelium in COVID-19 has been observed in kidneys, liver, heart, and intestine ${ }^{35}$. Additionally, an important feature of coronavirus disease-associated 
coagulopathy is the microcirculatory endothelial damage in the pulmonary circulation and other vascular beds. Since SARS-CoV-2 directly infects the vascular endothelial bed causing cellular damage and apoptosis ${ }^{36}$, the antithrombotic activity of the luminal surface is remarkably diminished ${ }^{37}$. On top of that, it is known that normal endothelial function denotes the capacity to regulating vascular tonus, permeability, cell adhesion, and anticoagulation. In COVID-19, the infected endothelial cells lose their ability to preserve the aforementioned physiological functions resulting in inflammation and the generation of a prothrombotic milieu with the formation of thrombosis, and organ malcirculation ${ }^{38}$. This infection-mediated endothelial injury (characterized by elevated levels of von Willebrand factor) and endothelialitis (marked by the presence of activated neutrophils and macrophages), found in multiple vascular beds (including the lungs, kidney, heart, small intestine, and liver) ${ }^{35-39.40}$. Undeniably, injury to the endothelial barrier results in uncovering of subendothelial matrix components, of which collagen and tissue factor (TF) are considered to be the most active hemostatic components. Consequently, endothelial damage can trigger the activation of platelets (e.g., via the collagen receptor glycoprotein VI) and the TF-dependent generation of thrombin and fibrin (via the extrinsic coagulation pathway of factor VII). It has been proposed that macrophages are the crucial cells in inducing thrombotic phenomena. It is known that macrophage activation, as well as macrophage viral infection itself, determines the production of immunomodulation cytokines and reactive oxygen species (ROS) from the early stages of the infection ${ }^{41}$.

Usually, macrophages have been presumed to be the basis of the majority of the ROS in the vessel walls, and unquestionably these cells show a vital role in vessel pathology 42. Macrophages infected with severe acute respiratory syndrome coronavirus 2 (SARS-Cov-2) produce the procoagulant prothrombinase, which is chief for both the initiation and localization of fibrin deposition ${ }^{43}$.

Platelet-neutrophil cross-communication and activation of macrophages in this setting can ease a variety of proinflammatory effects, such as cytokine release, the formation of neutrophil extracellular traps (NETs), and fibrin and/or microthrombus formation ${ }^{44,45}$. NETs further harm the endothelium and trigger both extrinsic coagulation pathways and intrinsic coagulation pathways. They were noticed at higher levels in patients hospitalized with COVID-19 in a study from a large academic center in the USA ( 50 patients and 30 control participants), with a 'proNETotic state' positively correlating with severe illness ${ }^{46}$. Hypoxia-mediated hyperviscosity and upregulation of the hypoxia-inducible factor-1 (HIF-1) signaling pathway after acute lung injury may also subsidize the prothrombotic ${ }^{47}$. Lastly, direct coronavirus-mediated effects may also lead to an imbalance of pro-and anti-coagulant pathways ${ }^{48,49}$.

Treatment options of COVID-19 coagulopathy.

As a consequence of an elevated risk of venous thromboembolism (VTE) in all patients admitted with a confirmed diagnosis or assumed COVID-19. All of them should be given pharmacological VTE prophylaxis without there are absolute contraindication ${ }^{50-51}$. In all patients with COVID-19, VTE risk should be weighed using a risk assessment model validated for acute medical illness. The VTE risk stratification should be repeated along with bleeding risk assessment during illness. On one hand, As said by the international society of thrombosis and hemostasis (ISTH), all patients, (including non-critically ill) who require hospital admission for COVID-19 infection should take prophylactic dose LMWH unless they have contra-indications (active bleeding and platelet count $\left.<25 \times 10^{9} / \mathrm{L}\right){ }^{52}$. Besides, The American Society of Hematology suggests that hospitalized patients with COVID-19 should receive pharmacologic thromboprophylaxis with Low-molecular-weight heparins (LMWH) or fondaparinux (preferred to unfractionated heparin (UFH) to reduce contact) unless there is increased bleeding risk.

If anticoagulants are unreachable or contraindicated, the use of mechanical thromboprophylaxis (e.g. pneumatic compression devices) is very crucial. Extremely ill COVID19 patients should not receive a therapeutic dose of anticoagulants empirically (i.e. in the absence of confirmed venous thromboembolism) ${ }^{53}$. According to the route of administration, LMWH was given subcutaneously once or twice daily. If LMWH is not available, unfractionated heparin could be used, although this requires more frequent injections ${ }^{54}$. Likewise, it is optional that, anticoagulant thromboprophylaxis with LMWH, fondaparinux, or UFH over anticoagulant thromboprophylaxis with a direct oral anticoagulant (DOAC) and LMWH and fondaparinux over UFH to limit staff exposure. Some against the use of DOACs as many hospitalized COVID-19 patients will be receiving concomitant therapy (antiviral agents) that can significantly affect the pharmacodynamics of and thus bleeding risk associated with the DOACs ${ }^{51}$. In the same way, the Italian Society of Thrombosis and Hemostasis (SISET) strongly restated that COVID-19 patients should be covered by LMWH, UFH, or fondaparinux at doses indicated for the prophylaxis of VTE for the entire duration of the hospital stay, and for 7-14 days more after hospital release ${ }^{55}$. Most of the foremost international guidelines and recommendations (ISTH-IG, ACF, CDC, and ASH) advise stopping anticoagulation in patients who are vigorously bleeding or severely thrombocytopenic. ISTH-IG acclaims stopping LMWH prophylactic anticoagulation for patients with platelet count less than $25 \times 10^{9} / \mathrm{L}$. ASH remarks that therapeutic anticoagulation should be held if platelet count $<30-50 \times 10^{9} / \mathrm{L}$ or fibrinogen $<1.0 \mathrm{~g} / \mathrm{L}$ and prophylactic anticoagulation should be stopped only if platelet count $<25 \times 10^{9} / \mathrm{L}$ or fibrinogen $<0.5 \mathrm{~g} / \mathrm{L}$. The SCC-ISTH does not recommend a particular platelet level to hold anticoagulation but does report that $50 \%$ of their respondents report holding for a platelet count $<25 \times 10^{9} / \mathrm{L}$. The CDC and ACF commend stopping prophylactic anticoagulation in severely thrombocytopenic patients, they also do not recommend a particular platelet count threshold. 
The ACCP does not make any specific recommendations concerning the holding of anticoagulation. The ACC mentions that in patients with moderate or severe COVID19 on chronic therapeutic anticoagulation who develop suspected or confirmed DIC with overt bleeding, it is advised to consider the indication of anticoagulation and risk of bleeding for adjusting dose or discontinuation of anticoagulation. The mainstream of their author's acclaim reducing the intensity of anticoagulation unless there is an exceedingly high risk of thrombosis. Worth mentioning, the ISTH-IG, ACF, and ASH mention that abnormal PT or PTT is not a contraindication to thromboprophylaxis 56 . Substantially, since the prothrombotic role of the cytokine storm and of the possibility of SARS-CoV2 to induce high IL-6 levels and complement cascade activation contributing to the prothrombotic state of COVID-19 patients, the use of anti-inflammatory drugs as corticosteroids and hMAb that target and inhibit the IL-6 pathway and complement cascade activation (Tocilizumab and Eculizumab, respectively) may be convenient ${ }^{57}$.

In the same regard, The National Institutes of Health (NIH) does not mention routine post-discharge VTE prophylaxis for patients with COVID-19 (level of evidence: AIII-strong, based on expert opinion). However, based on benefits of post-discharge prophylaxis for certain high-risk patients without COVID-19, the Food and Drug Administration (FDA) approved two regimens of direct oral anticoagulants: Rivaroxaban $10 \mathrm{mg}$ daily for 31 to 39 days, and betrixaban $160 \mathrm{mg}$ on day 1 , followed by $80 \mathrm{mg}$ once daily for 35 to 42 days 58 .

All in all, dynamic interrogations endure to be talked, thorough the following:

1. Must all hospitalized patients with severe SARSCov-2 with a respiratory decline and/or hemodynamic variability be treated with anticoagulation therapy until the diagnosis of VTE is obtainable?

2. Had better thromboprophylaxis been nonstop after hospital release?

3. Do the available data of the specific types such as unfractionated heparin or low-molecular-weight heparin $(\mathrm{LMWH})$ ) or LMWH derivatives like enoxaparin, dalteparin, or tinzaparin, have unraveled a good role for choosing between highrisk patients?

4. Concerning VTE, Should the patient have the right to take thromboprophylaxis, especially if have a high risk for VTE after recovery from COVID-19 or hospital discharge?

\section{Conclusion}

SARS-CoV-2 causing COVID-19 has diseased a large number of people all over the world. Contagion with this disease has an exclusive laboratory signature with thrombocytopenia through raised fibrinogen/fibrin and Ddimer, all of which are connected with poor consequences. Thrombotic outcomes add to morbidity and mortality and necessitate a thoughtful tactic to VTE prophylaxis, harmonizing the risk of thrombosis with the risk of major bleeding. Last but not least, we await upcoming trials that enable the formal progress of rigorous clinical practice guidelines in this regard.

References

1. Huang, C., Wang, Y., Li, X., et al. (2020). Clinical features of patients infected with 2019 novel coronavirus in Wuhan, China. The lancet, 395(10223), 497-506.

2. Guan, W. J., Ni, Z. Y., Hu, Y., et al. (2020). Clinical characteristics of coronavirus disease 2019 in China. New England journal of medicine, 382(18), 1708-1720.

3. Zhou, F., Yu, T., Du, R., et al. (2020). Clinical course and risk factors for mortality of adult inpatients with COVID-19 in Wuhan, China: a retrospective cohort study. The lancet, 395(10229), 1054-1062.

4. Shiha, G., Mousa, N., Ahmed Abdel-Razik A., et al. (2020). COVID-19: More Than A Lung Infection. Clinics of Oncology, 3(3): 1-8.

5. Zou, X., Chen, K., Zou, J., et al. (2020). Single-cell RNA-seq data analysis on the receptor ACE2 expression reveals the potential risk of different human organs vulnerable to 2019-nCoV infection. Frontiers of medicine, 1-8.

6. Xu, X., Chen, P., Wang, J., et al. (2020). Evolution of the novel coronavirus from the ongoing Wuhan outbreak and modeling of its spike protein for risk of human transmission. Science China Life Sciences, 63(3), 457-460.

7. Connors, J. M., and Levy, J. H. (2020). Thromboinflammation and the hypercoagulability of COVID-19. Journal of Thrombosis and Haemostasis, 18(7), 1559-1561.

8. Thachil, J., Cushman, M., and Srivastava, A. (2020). A proposal for staging COVID-19 coagulopathy. Research and practice in thrombosis and haemostasis, 4(5), 731-736.

9. Mehta, P., McAuley, D. F., Brown, M., et al. (2020). COVID-19: consider cytokine storm syndromes and immunosuppression. The lancet, 395(10229), 10331034.

10. Connors, J. M., and Levy, J. H. (2020). COVID-19 and its implications for thrombosis and anticoagulation. Blood, The Journal of the American Society of Hematology, 135(23), 2033-2040.

11. Klok, F. A., Kruip, M. J. H. A., Van der Meer, N. J. M., et al. (2020). Incidence of thrombotic complications in critically ill ICU patients with COVID-19. Thrombosis research, 191, 145-147.

12. Tang, N., Li, D., Wang, X., et al. (2020). Abnormal coagulation parameters are associated with poor prognosis in patients with novel coronavirus pneumonia. Journal of thrombosis and haemostasis, 18(4), 844-847.

13. Chen, J., Wang, X., Zhang, S., et al. (2020). Findings of acute pulmonary embolism in COVID-19 patients. Available at SSRN 3548771. 
14. Poor, H. D., Ventetuolo, C. E., Tolbert, T., et al. (2020). COVID-19 critical illness pathophysiology driven by diffuse pulmonary thrombi and pulmonary endothelial dysfunction responsive to thrombolysis. Clinical and translational medicine, 10(2), e44.

15. Cui, S., Chen, S., Li, X., et al. (2020). Prevalence of venous thromboembolism in patients with severe novel coronavirus pneumonia. Journal of Thrombosis and Haemostasis, 18(6), 1421-1424.

16. Miesbach, W., and Makris, M. (2020). COVID-19: coagulopathy, risk of thrombosis, and the rationale for anticoagulation. Clinical and Applied Thrombosis/Hemostasis, 26, 1076029620938149.

17. Zhou, F., Yu, T., Du, R., et al. (2020). Clinical course and risk factors for mortality of adult inpatients with COVID-19 in Wuhan, China: a retrospective cohort study. The lancet, 395(10229), 1054-1062.

18. Tang, N., Li, D., Wang, X., et al. (2020). Abnormal coagulation parameters are associated with poor prognosis in patients with novel coronavirus pneumonia. Journal of thrombosis and haemostasis, 18(4), 844-847.

19. Robba, C., Battaglini, D., Ball, L., et al. (2021). Coagulative Disorders in Critically Ill COVID-19 Patients with Acute Distress Respiratory Syndrome: A Critical Review. Journal of Clinical Medicine, 10(1), 140.

20. Garcia-Olivé, I., Sintes, H., Radua, J., et al. (2020). D-dimer in patients infected with COVID-19 and suspected pulmonary embolism. Respiratory medicine, 169, 106023.

21. Zhang, L., Yan, X., Fan, Q., et al. (2020). D-dimer levels on admission to predict in-hospital mortality in patients with Covid-19. Journal of Thrombosis and Haemostasis, 18(6), 1324-1329.

22. Tang, N., Bai, H., Chen, X., et al. (2020). Anticoagulant treatment is associated with decreased mortality in severe coronavirus disease 2019 patients with coagulopathy. Journal of thrombosis and haemostasis, 18(5), 1094-1099.

23. Thachil, J., Tang, N., Gando, S., et al. (2020). ISTH interim guidance on recognition and management of coagulopathy in COVID-19. Journal of Thrombosis and Haemostasis, 18(5), 1023-1026.

24. Bosevski, M., Krstevski, G., Bosevska, G., et al. (2021). The role of D-dimer in relation to the clinical course of patients with COVID-19. Acta Biochimica et Biophysica Sinica, 53(1), 119-120.

25. Marietta, M., Ageno, W., Artoni, A., et al. (2020). COVID-19 and haemostasis: a position paper from Italian Society on Thrombosis and Haemostasis (SISET). Blood Transfusion, 18(3), 167.

26. Liu, Y., Yang, Y., Zhang, C., et al. (2020). Clinical and biochemical indexes from 2019-nCoV infected patients linked to viral loads and lung injury. Science China Life Sciences, 63(3):364-374.

27. Du, L., Zhao, G., He, Y., et al. (2007). Receptorbinding domain of SARS-CoV spike protein induces long-term protective immunity in an animal model. Vaccine, 25(15), 2832-2838.

28. Li, W., Greenough, T. C., Moore, M. J., et al. (2004). Efficient replication of severe acute respiratory syndrome coronavirus in mouse cells is limited by murine angiotensin-converting enzyme 2. Journal of virology, 78(20), 11429-11433.

29. Li, W., Moore, M. J., Vasilieva, N., et al. (2003). Angiotensin-converting enzyme 2 is a functional receptor for the SARS coronavirus. Nature, 426(6965), 450-454.

30. Xiao, X., Chakraborti, S., Dimitrov, A. S., et al. (2003). The SARS-CoV S glycoprotein: expression and functional characterization. Biochemical and biophysical research communications, 312(4), 11591164.

31. Hoffmann, M., Kleine-Weber, H., Schroeder, S., et al. (2020). SARS-CoV-2 cell entry depends on ACE2 and TMPRSS 2 and is blocked by a clinically proven protease inhibitor. cell, 181(2), 271-280.

32. Wrapp, D., Wang, N., Corbett, K. S., et al. (2020). Cryo-EM structure of the 2019-nCoV spike in the prefusion conformation. Science, 367(6483), 12601263.

33. Bray, M. A., Sartain, S. A., Gollamudi, J., et al. (2020). Microvascular thrombosis: experimental and clinical implications. Translational Research. 1

34. Leisman, D. E., Deutschman, C. S., and Legrand, M. (2020). Facing COVID-19 in the ICU: vascular dysfunction, thrombosis, and dysregulated inflammation. Intensive care medicine, 46(6), 11051108.

35. Varga, Z., Flammer, A. J., Steiger, P., et al. (2020). Endothelial cell infection and endotheliitis in COVID-19. The Lancet, 395(10234), 1417-1418.

36. Gustafson, D., Raju, S., Wu, R., et al. (2020). Overcoming barriers: the endothelium as a linchpin of coronavirus disease 2019 pathogenesis? Arteriosclerosis, thrombosis, and vascular biology, 40(8), 1818-1829.

37. Wichmann, D., and Sperhake, J. P. (2020). Lütgehetmann Met al. Autopsy findings and venous thromboembolism in patients with COVID-19: a prospective cohort study Ann Intern Med2020. Doi, 10, M20-2003.

38. Iba, T., Connors, J. M., and Levy, J. H. (2020). The coagulopathy, endotheliopathy, and vasculitis of COVID-19. Inflammation Research, 1-9.

39. Iba, T., and Levy, J. H. (2018). Inflammation and thrombosis: roles of neutrophils, platelets and endothelial cells and their interactions in thrombus formation during sepsis. Journal of Thrombosis and Haemostasis, 16(2), 231-241.

40. Merad, M., and Martin, J. C. (2020). Pathological inflammation in patients with COVID-19: a key role for monocytes and macrophages. Nature Reviews Immunology, 20(6), 355-362.

41. Cathcart, M. K. (2004). Regulation of superoxide anion production by NADPH oxidase in 


\section{Review Article}

monocytes/macrophages: contributions to atherosclerosis. Arteriosclerosis, thrombosis, and vascular biology, 24(1), 23-28.

42. Dandekar, A. A., and Perlman, S. (2005). Immunopathogenesis of coronavirus infections: implications for SARS. Nature reviews immunology, 5(12), 917-927.

43. Giannis, D., Ziogas, I. A., and Gianni, P. (2020). Coagulation disorders in coronavirus infected patients: COVID-19, SARS-CoV-1, MERS-CoV and lessons from the past. Journal of Clinical Virology, 127, 104362.

44. Koupenova, M., Corkrey, H. A., Vitseva, O., et al. (2019). The role of platelets in mediating a response to human influenza infection. Nature communications, 10(1), 1-18.

45. Zuo, Y., Zuo, M., Yalavarthi, S., et al. (2020). Neutrophil extracellular traps and thrombosis in COVID-19. Journal of thrombosis and thrombolysis, 1-8.

46. Gupta, N., Zhao, Y. Y., and Evans, C. E. (2019). The stimulation of thrombosis by hypoxia. Thrombosis research, 181, 77-83.

47. Giannis, D., Ziogas, I. A., and Gianni, P. (2020). Coagulation disorders in coronavirus infected patients: COVID-19, SARS-CoV-1, MERS-CoV and lessons from the past. Journal of Clinical Virology, 127, 104362.

48. Deshpande, C. (2020). Thromboembolic findings in COVID-19 autopsies: pulmonary thrombosis or embolism? Annals of internal medicine, 173(5), 394395.

49. Schünemann, H. J., Cushman, M., Burnett, A. E., et al. (2018). American Society of Hematology 2018 guidelines for management of venous thromboembolism: prophylaxis for hospitalized and nonhospitalized medical patients. Blood advances, 2(22), 3198-3225.

50. Tal, S., Spectre, G., Kornowski, R., and Perl, L. (2020). Venous thromboembolism complicated with COVID-19: what do we know so far? Acta haematologica, 143(5), 417-424.

51. Moores, L. K., Tritschler, T., Brosnahan, S., et al. (2020). Prevention, diagnosis and treatment of venous thromboembolism in patients with COVID19: CHEST Guideline and Expert Panel Report. Chest.

52. Thachil, J., Tang, N., Gando, S., et al. (2020). ISTH interim guidance on recognition and management of coagulopathy in COVID-19. Journal of Thrombosis and Haemostasis, 18(5), 1023-1026.

53. Atallah, B., Mallah, S. I., and AlMahmeed, W. (2020). Anticoagulation in COVID-19.

54. Zhai, Z., Li, C., Chen, Y., et al. (2020). Prevention and treatment of venous thromboembolism associated with coronavirus disease 2019 infection: a consensus statement before guidelines. Thrombosis and haemostasis, 120(6), 937.
55. Marietta, M., Ageno, W., Artoni, A., et al. (2020). COVID-19 and haemostasis: a position paper from Italian Society on Thrombosis and Haemostasis (SISET). Blood Transfusion, 18(3), 167.

56. Barrett, C. D., Moore, H. B., Yaffe, M. B., et al. (2020). ISTH interim guidance on recognition and management of coagulopathy in COVID-19: A Comment. Journal of Thrombosis and Haemostasis, 18(8), 2060-2063.

57. Marfella, R., Paolisso, P., Sardu, C., et al. (2020). Negative impact of hyperglycaemia on tocilizumab therapy in Covid-19 patients. Diabetes \& metabolism, 46(5), 403-405.

58. Abdelnabi, M., Leelaviwat, N., Eshak, N., et al. (2021, January). COVID-19 discharge and follow-up recommendations. In Baylor University Medical Center Proceedings (Vol. 34, No. 1, pp. 73-75). Taylor \& Francis. 